\title{
On the dispersion of two coexisting nongyrotropic ion species
}

\author{
A. L. Brinca ${ }^{1}$, U. Motschmann ${ }^{2}$, F. J. Romeiras ${ }^{1}$ \\ ${ }^{1}$ Centro de Fisica de Plasmas, Instituto Superior Técnico, P-1096 Lisbon Codex, Portugal \\ ${ }^{2}$ Institut für Theoretische Physik, Technische Universität Braunschweig, D-38106 Braunschweig, Germany
}

Received: 21 October 1998 / Revised: 8 March 1999 / Accepted: 16 March 1999

\begin{abstract}
Space observations in the solar wind and simulations of high Mach number bow-shocks have detected particle populations with two coexisting nongyrotropic ion species. We investigate the influence of these two sources of free energy on the stability of parallel (with respect to the ambient magnetic field) and perpendicular propagation. For parallel modes, we derive their dispersion equation in a magnetoplasma with protons and alpha particles that may exhibit stationary nongyrotropy (SNG) and discuss the characteristics of its solutions. Kinetic simulations study the behaviour of perpendicular electrostatic (Bernstein-like) waves in a plasma whose ion populations (positrons and fictitious singly-charged particles with twice the electron mass, for the sake of simulation feasability) can be timevarying nongyrotropic (TNG). The results show that the coexistence of two gyrophase bunched species does not significantly enhance the parallel SNG instability already found for media with only one nongyrotropic species, whereas it strongly intensifies the growth of Bernstein-like modes in TNG plasmas.
\end{abstract}

Key words. Magnetospheric physics (plasma waves and instabilities) - Space plasma physics (numerical simulation studies; waves and instabilities)

\section{Introduction}

The frequent observation of nongyrotropic particle species in space plasmas (i.e. populations whose unperturbed distributions exhibit some degree of gyrophase organization) has motivated several studies on their stability (references for these works on observation and stability of nongyrotropy can be found in the papers

Correspondence to: A. L. Brinca

e.mail: ebrinca@beta.ist.utl.pt cited). In homogeneous media, two models of nongyrotropy have been explored: closed phase spaces with unperturbed rotating time-varying distributions (TNG) and open phase spaces (with sources and sinks) with stationary equilibrium distributions (SNG). Both environments can bring about for parallel propagation (with respect to the ambient magnetic field) linear coupling among the eigenwaves of the associated gyrotropic system (longitudinal electrostatic, tranverse electromagnetic left- and right-hand circularly polarized modes), enhance previously existing instabilities, and destabilize otherwise thoroughly passive magnetoplasmas. For perpendicular (oblique, in general) propagation the nongyrotropic studies are scarcer, but they also demonstrate the capability of gyrophase bunching to feed wave growth in both TNG and SNG environments. All these results have been derived for media with only one nongyrotropic species and, in the case of the SNG studies, assuming that the sources and sinks of that species were not influenced by the wave perturbations (sources and sinks "external" to the system).

Here, motivated by (rarer and more recent) observations of different coexisting nongyrotropic populations [Astudillo et al. (1996) report alpha particles and protons with gyrophase organization in the solar wind; Manfred Scholer (private communication, 1998) has detected coexisting populations of gyrophase bunched protons and alpha particles in simulations of high Mach number bow-shocks], we (i) extend previous investigations of the SNG stability of parallel propagation to this situation, cast the results in a form that can be directly generalized to an arbitrary number of nongyrotropic species, adopt a very simple model to illustrate the (strongly stabilizing) effects of "internal" sinks on the complex dispersion of SNG systems, and (ii) use kinetic simulations to assess the role of two coexisting TNG ion species (positrons and singly positively charged particles with twice the electron mass) on the perpendicular propagation of electrostatic Bernstein-like waves.

The investigation shows that whereas the consequences of the coexistence of two nongyrotropic pop- 
ulations can be qualitatively anticipated for parallel SNG stability from the situations encountered with only one gyrophase bunched species, the combined effects of two nongyrotropic ion populations (with harmonically related cyclotron frequencies) provide a very strong enhancement in the perpendicular electrostatic wave growth.

\section{Parallel propagation}

For the study of the parallel stability, we consider a neutral, parallel-current free, homogeneous magnetoplasma with an ambient magnetic field $\mathbf{B}_{o}=B_{o} \hat{\mathbf{x}}$ and five particle populations whose parameters are identified by the subscripts $e$ (electrons), $p$ (gyrotropic protons), $a$ (gyrotropic alpha particles), $b$ (nongyrotropic protons), and $d$ (nongyrotropic alpha particles). Denoting the unperturbed number densities and parallel drift velocities by $N_{j}$ and $V_{D j}(j=e, p, a, b, d)$, respectively, one should thus satisfy $N_{e}=N_{p}+N_{b}+2\left(N_{a}+N_{d}\right)$ and $N_{e} V_{D e}=N_{p} V_{D p}+N_{b} V_{D b}+2\left(N_{a} V_{D a}+N_{d} V_{D d}\right)$. The angular cyclotron and plasma frequencies of species $j$ are defined by

$\Omega_{j}=\frac{q_{j} B_{o}}{m_{j}} \quad$ and $\quad \omega_{p j}^{2}=\frac{N_{j} q_{j}^{2}}{\epsilon_{o} m_{j}}$

where $q_{e}=-e$ and the remaining notation is standard.

With the attention focussed in parallel propagation, the wavevector satisfies $\mathbf{k}=k \hat{\mathbf{x}}$ and only the spatial coordinate $x$ enters the analysis. We adopt cylindrical coordinates in velocity space $\left(v_{x}, v_{\perp}, \varphi\right)$ where the gyrophase is defined by $\varphi=\arctan \left(v_{z} / v_{y}\right)$.

\subsection{Gyrotropic medium}

The gyrotropic species $(l=e, p, a)$ have equilibrium distributions $F_{o l}=F_{o l}\left(v_{x}, v_{\perp}\right)$ that can be particularized to (possibly drifting and anisotropic) Maxwellians

$$
\begin{aligned}
F_{o l} & =M_{l}\left(v_{t l}, V_{D l}, A_{l}\right) \\
& =\frac{\exp \left[-v_{\perp}^{2} /\left(A_{l} v_{t l}^{2}\right)\right]}{A_{l}\left(\sqrt{\pi} v_{t l}\right)^{3}} \exp \left[-\left(v_{x}-V_{D l}\right)^{2} / v_{t l}^{2}\right]
\end{aligned}
$$

with temperature anisotropies $A_{l}=T_{\perp l} / T_{x l}$ and parallel thermal velocities $v_{t l}=\left(2 T_{x l} / m_{j}\right)^{1 / 2}$.

\subsection{SNG ions}

In homogeneous media with closed phase spaces (particle distribution functions obey homogeneous Vlasov equations), the unperturbed (zero wave fields) distributions of the nongyrotropic species must be timevarying (TNG media). We shall be concerned here with open phase spaces (source, $S$, and sink, $L$, terms on the rhs of the Vlasov equation) where the unperturbed nongyrotropic distribution can be homogeneous and stationary (SNG environments) as shown, for example, in Motschmann et al. (1997). Furthermore, we shall assume that the sources and sinks of the nongyrotropic populations are not affected by the small amplitude wave perturbations so that their linearization, ()$=()_{o}+()_{1}, \quad$ yields $\quad S=S_{o} \quad$ and $\quad L=L_{o}$ $\left(S_{1}=L_{1}=0\right)$; they are "external" to the system. In Appendix B we shall analyze and comment upon the effects associated with simple BGK-like (proportional to the nongyrotropic distribution functions) "internal" loss terms $\left(L_{1} \neq 0\right)$.

Following the approach adopted in Brinca and Romeiras (1998) for "external" SNG environments, we assume the stationary unperturbed distributions of the nongyrotropic ions $(s=b, d)$ take the separable form

$F_{o s}=G_{o s}\left(v_{x}, v_{\perp}\right) \Phi_{s}(\varphi)$

with

$$
\begin{aligned}
& \Phi_{s}(\varphi)=\sum_{n=-\infty}^{\infty} \phi_{s n} e^{-i n \varphi}, \quad \phi_{s n}=\frac{1}{2 \pi} \int_{2 \pi} \Phi_{s}(\varphi) e^{i n \varphi} \mathrm{d} \varphi, \\
& \Phi_{s} \geq 0, \quad \phi_{s n}=\phi_{s(-n)}^{*}, \quad \int_{2 \pi} \Phi_{s}(\varphi) \mathrm{d} \varphi=1=2 \pi \phi_{s o}
\end{aligned}
$$

and, when their dependence on $\left(v_{x}, v_{\perp}\right)$ is assumed Maxwellian,

$G_{o s}\left(v_{x}, v_{\perp}\right)=2 \pi M_{s}\left(v_{t s}, V_{D s}, A_{s}\right)$

\subsection{Wave and dispersion equations}

The original approach adopted in the study of parallel wave dispersion in nongyrotropic magnetoplasmas was established by Sudan (1965) for homogeneous timevarying unperturbed nongyrotropic (TNG) populations. The behaviour of the system is governed by the Maxwell and Vlasov equations: their linearization and compatibilization yields a matrix wave equation whose nontrivial solutions require satisfaction of the dispersion equation.

More recently, several space observations suggested that there exist instances where the SNG model is more adequate. The ensuing analyses of the stability of stationary nongyrotropy (Cao et al., 1995; Motschmann et al., 1997; Brinca and Romeiras, 1998; Cao et al., 1998; Motschmann and Glaßmeier, 1998) were performed for a single SNG species. Here, with the motivation described in the introduction, we study the dispersion characteristics of a SNG magnetoplasma with two (protons and alpha particles) nongyrotropic populations. The derivation of the relevant wave and dispersion equations for this case is an extension and adaptation of the procedure used for the single SNG population. As in Brinca and Romeiras (1998), we assume that the source and sink terms in the Vlasov equation do not depend on the wave perturbations under study (the SNG populations are "externally" driven). The structure of the matrix wave equation is independent of the number of SNG species, 


$$
\left(\begin{array}{ccc}
m_{++} & m_{+x} & m_{+-} \\
m_{x+} & m_{x x} & m_{x-} \\
m_{-+} & m_{-x} & m_{--}
\end{array}\right)\left(\begin{array}{c}
\bar{E}_{+} \\
\bar{E}_{x} \\
\bar{E}_{-}
\end{array}\right)=0,
$$

but the matrix elements defined in Appendix A contain the contributions of all (gyrotropic and nongyrotropic populations); $\bar{E}_{\eta}$ stands for the complex amplitudes of the wave electric field components with

()$_{ \pm}=\left[()_{y} \pm i()_{z}\right] / 2$.

Nontrivial field solutions imply satisfaction of the dispersion equation

$\operatorname{det}\left(m_{\eta v}\right)=D\left(\omega=\omega_{r}+i \omega_{i}, k\right)=0$.

The qualitative properties of the $\mathrm{SNG}$ dispersion encountered for a single nongyrotropic species are recovered here. In particular, recalling that $m_{\eta \eta}=0$ yields the dispersion equations of the gyrotropic parallel eigenmodes $(\eta=+$, left-hand electromagnetic, $\eta=x$, electrostatic, $\eta=-$, right-hand electromagnetic) and that the off-diagonal terms originate in the SNG populations, we notice that the introduction of nongyrotropy creates coupling among the parallel eigenwaves: these interactions are mediated by the nongyrotropic particles. The consequences of the coupling depend on the parameters of the medium, with the unperturbed nongyrotropic distributions influencing the linear wave dispersion through the values of the first coefficients of their Fourier expansion $\phi_{s n}(|n| \leq 2, s=b, d)$. Coupling of the electrostatic eigenmode to the electromagnetic eigenwaves requires the existence of a finite unperturbed perpendicular current $\left(\phi_{s( \pm 1)} \neq 0\right.$, $s=b$ and, or $s=d$ ) whereas $\phi_{s( \pm 2)} \neq 0$ brings about interaction between the transverse electromagnetic modes.

Specialization of the unperturbed (gyrotropic and nongyrotropic) particle distributions to (possibly drifting and anisotropic) Maxwellians allows for the calculation of the matrix elements $m_{\eta v}$, yielding

$$
\begin{aligned}
m_{++}= & k^{2} c^{2}-\omega^{2}-\sum_{j=e, p, a, b, d} \omega_{p j}^{2} \\
& \times\left[\frac{\omega}{k v_{t j}}\left(1-\frac{k V_{D j}}{\omega}\right) Z\left(\xi_{j+}\right)-\frac{1}{2}\left(A_{j}-1\right) Z^{\prime}\left(\xi_{j+}\right)\right] \\
m_{+x}= & \frac{\pi^{3 / 2}}{2} \sum_{s=b, d} \sqrt{A_{s}} \frac{\omega}{k v_{t s}} \omega_{p s}^{2} \phi_{s(+1)} Z^{\prime}\left(\xi_{s+}\right) \\
m_{+-}= & \pi \sum_{s=b, d} A_{s} \omega_{p s}^{2} \phi_{s(+2)} Z^{\prime}\left(\xi_{s+}\right) \\
m_{x+}= & -\pi^{3 / 2} \sum_{s=b, d} \sqrt{A_{s}} \frac{\omega_{p s}^{2}}{\omega k v_{t s}} \phi_{s(-1)} Z^{\prime}\left(\xi_{s x}\right) \\
m_{x x}= & 1-\sum_{j=e, p, a, b, d}\left(\frac{\omega_{p j}}{k v_{t j}}\right)^{2} Z^{\prime}\left(\xi_{j x}\right) \\
m_{x-}= & -\pi^{3 / 2} \sum_{s=b, d} \sqrt{A_{s}} \frac{\omega_{p s}^{2}}{\omega k v_{t s}} \phi_{s(+1)} Z^{\prime}\left(\xi_{s x}\right)
\end{aligned}
$$

$$
\begin{aligned}
m_{-+}= & \pi \sum_{s=b, d} A_{s} \omega_{p s}^{2} \phi_{s(-2)} Z^{\prime}\left(\xi_{s-}\right) \\
m_{-x}= & \frac{\pi^{3 / 2}}{2} \sum_{s=b, d} \sqrt{A_{s}} \frac{\omega}{k v_{t s}} \omega_{p s}^{2} \phi_{s(-1)} Z^{\prime}\left(\xi_{s-}\right) \\
m_{--}= & k^{2} c^{2}-\omega^{2}-\sum_{j=e, p, a, b, d} \omega_{p j}^{2} \\
& \times\left[\frac{\omega}{k v_{t j}}\left(1-\frac{k V_{D j}}{\omega}\right) Z\left(\xi_{j-}\right)-\frac{1}{2}\left(A_{j}-1\right) Z^{\prime}\left(\xi_{j-}\right)\right]
\end{aligned}
$$

where

$\xi_{j \pm}=\frac{\omega-k V_{D j} \mp \Omega_{j}}{k v_{t j}} \quad \xi_{j x}=\frac{\omega-k V_{D j}}{k v_{t j}}$

and $Z(\xi)$ and $Z^{\prime}(\xi)$ represent the plasma dispersion function and its derivative.

\subsection{Complex dispersion}

Gyrotropic behaviour. The reference stable gyrotropic magnetoplasma used in the study of the wave dispersion in SNG media with coexisting nongyrotropic species has non-drifting isotropic Maxwellian populations of electrons, protons, and alpha particles with $B_{o}=5 n T$, $N_{e}=2 N_{p}=4 N_{a}=15.5 \mathrm{~cm}^{-3}, A_{e}=A_{p}=A_{a}=1, V_{D e}=$ $V_{D p}=V_{D a}=0, T_{e}=74 \mathrm{eV}$, and $T_{p}=T_{a}=5 \mathrm{eV}$, yielding $\beta_{e}=19.24, \quad \beta_{p}=0.65, \quad \beta_{a}=0.325$, and an Alfvén speed $v_{A}=B_{o}\left(\mu_{o} \sum N_{s} m_{s}\right)^{-1 / 2} \approx 22.6 \mathrm{~km} \mathrm{~s}^{-1}$. Its lowfrequency complex dispersion is shown in Fig. 1 for the eigenmodes of parallel propagation. The longitudinal electrostatic and transverse right-hand polarized electromagnetic modes exhibit the expected stable dispersion whereas the real dispersion of the left-hand wave deviates from the qualitative behaviour found in cold plasmas. Indeed, in cold magnetoplasmas with two species of positive ions, as is the case under consideration (protons and alpha particles), the real lowfrequency dispersion $\omega_{r}(k)$ of the parallel left-hand modes has two branches: the lower frequency one starts at the origin $\left(\omega_{r}=k=0\right)$ and goes into a resonance at $\omega_{r}=\Omega_{a}$ for large wavenumbers while the higher frequency branch evolves from a cutoff frequency $\omega_{c f}>\Omega_{a}$ to a resonance at $\Omega_{p}$ (for example, Mann et al. (1997)). The deviations from this cold dispersion of the left-hand mode in Fig. 1 are due to (finite) temperature effects encountered in the upper branch (no resonance occurs and the curve $\omega_{r}(k)$, started at $\omega_{r}=\omega_{c f}$, crosses $\omega_{r}=\Omega_{p}$ as $k$ increases) and in the lower branch (the resonance frequency $\Omega_{a}$ is not approached and, if the increase in $k$ were pursued farther, one would actually observe a gradual decrease of $\omega_{r}$ ); notice, however, that these deviations in the left-hand branches coexist with (very) large damping rates.

Nongyrotropic stability. To assess the destabilizing influence of the coexisting nongyrotropic populations of protons and alpha particles, we analyze extreme situations with respect to their density and gyrophase 

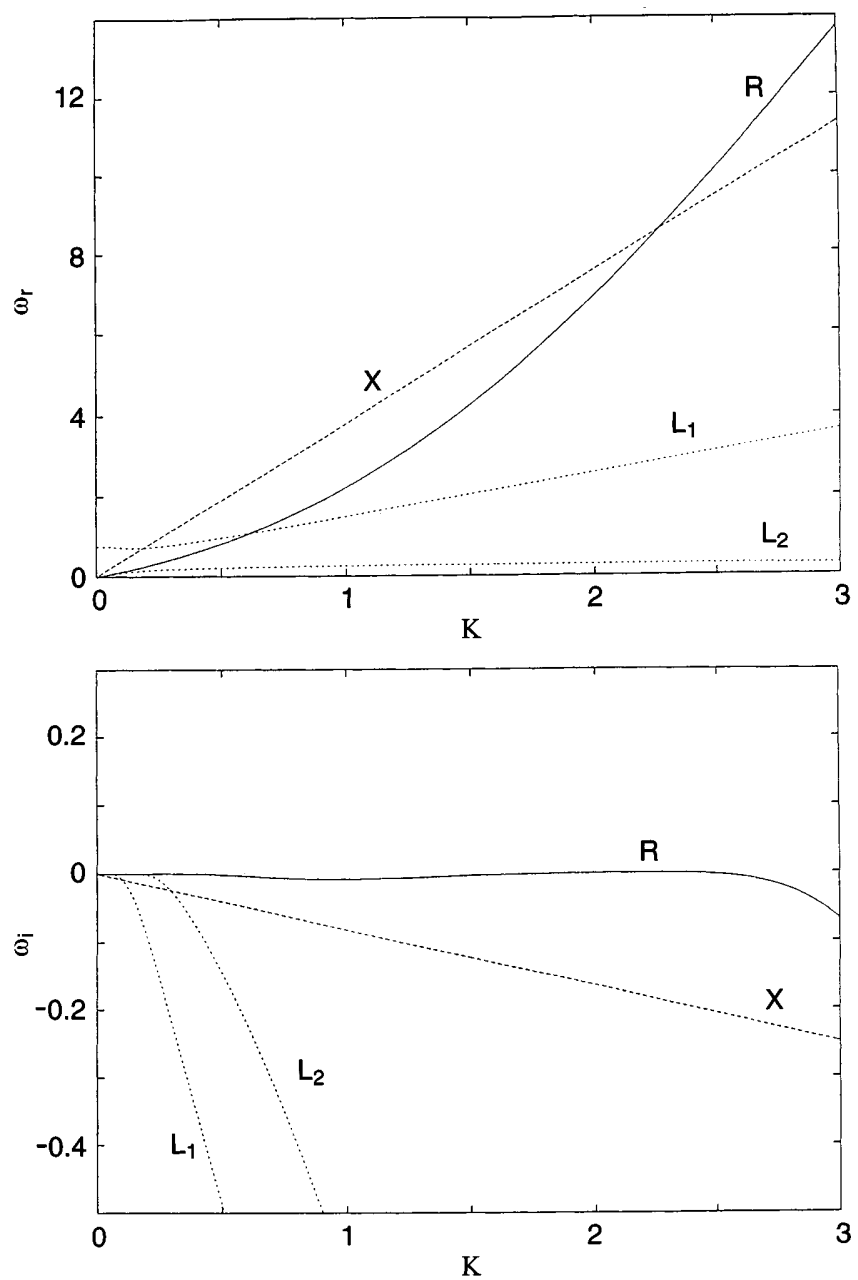

Fig. 1. Real and imaginary low-frequency dispersion of the parallel characteristic modes for the reference gyrotropic magnetoplasma: electrostatic $(X)$, electromagnetic right-hand $(R)$ and electromagnetic left-hand $\left(L_{1}\right.$ and $\left.L_{2}\right)$. Frequency and wavenumber are normalized to $\Omega_{p}$ and $\Omega_{p} / v_{A}$, respectively

distribution. We assume that nongyrotropy was introduced in all the ions $\left(N_{p}=N_{a}=0,2 N_{b}=4 N_{d}=N_{e}\right)$ of the (previously gyrotropic and stable) reference magnetoplasma and consider two possible types of $2 \pi$-periodic gyrophase organization: either

$\Phi_{s}(\varphi)=0, \quad-\pi \geq \varphi>\pi$

with the gyrophases of the ions sharing a common value (monochromatic gyrophases), or

$\Phi_{s}(\varphi)=\frac{1}{2}[\delta(\varphi)+\delta(\varphi-\pi)], \quad-\pi \geq \varphi>\pi$

with the gyrophases evenly distributed between two values differing by $\pi$ (dichromatic gyrophases). [As already pointed out in previous work (Brinca and Romeiras 1998), consideration of more realistic distributions should not modify the qualitative results obtained here.]

In the monochromatic case there occurs coupling among the (left- and right-hand circularly polarized) transverse electromagnetic eigenmodes and the longitudinal electrostatic mode. The complex dispersion for the resulting growing waves is displayed in Fig. 2: two hybrid (because of the interaction among the eigenwaves) modes have become unstable as a result of the introduction of these gyrophase bunchings in the ions of the previously thoroughly stable magnetoplasma. One of the modes is unstable within a wavenumber band bounded by $0.22<k v_{A} / \Omega_{p}<0.43$, reaching a maximum growth of $\omega_{i} / \Omega_{p}=0.015$ at $k v_{A} / \Omega_{p}=0.29$; the other growing mode is weakly unstable at small wavenumbers (maximum growth of $\omega_{i} / \Omega_{p}=0.0048$ at $\left.k v_{A} / \Omega_{p}=0.044\right)$ and is also unstable in the band $1.38<k v_{A} / \Omega_{p}<2.68$ with a maximum of $\omega_{i} / \Omega_{p}=0.225$ at $k v_{A} / \Omega_{p}=2.32$. As already stressed, these instabilities arise from the coupling, mediated by the nongyrotropic particles, of the eigenmodes of gyrotropic parallel propagation; for example, it becomes clear from comparison of Figs. 1 and 2 that the instability with the largest growth rate arises from the interaction (made possible by the nongyrotropic species, more specifically, by the existence of a nonzero first harmonic component in the gyrophase distributions of these species) between the gyrotropic modes labeled in
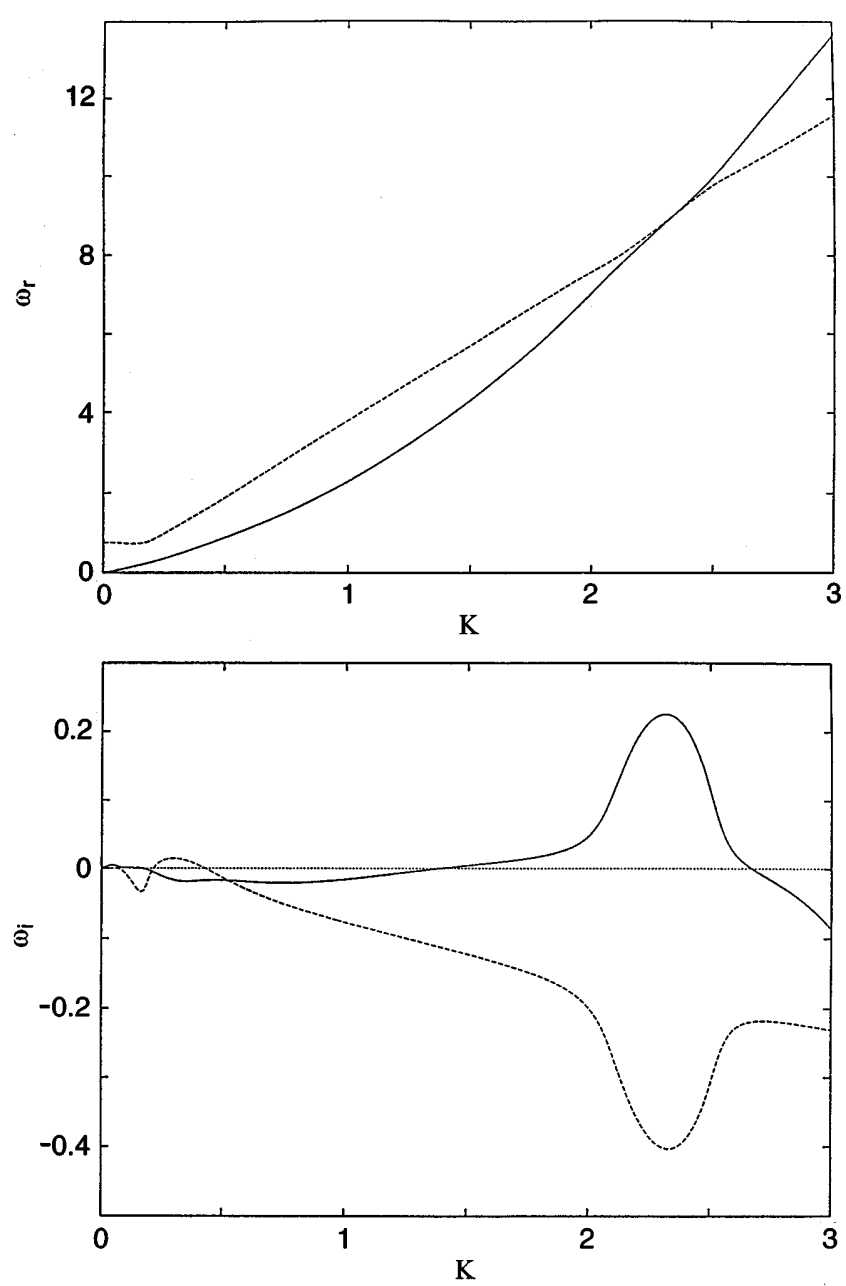

Fig. 2. Real and imaginary parallel dispersion of the reference magnetoplasma (unstable modes) when the unperturbed gyrophases of the protons and alpha particles are monochromatic. Normalizations as in Fig. 1. 
Fig. 1 by $\mathrm{X}$ (longitudinal electrostatic) and $\mathrm{R}$ (transverse electromagnetic right-hand circularly polarized). In order to identify the contributions of the two nongyrotropic species to the mode exhibiting two instabilities (solid line in Fig. 2), we analysed the complex dispersion of similar media where the monochromatic nongyrotropy occurred only in one species (that is, we either gyrotropized the alpha particles or the protons); as shown in Fig. 3, we found that both isolated nongyrotric species can feed two instabilities, albeit the nongyrotropic protons are the major contributors to the large wavenumber instability (large solid line bump in the lower panel of Fig. 2), and the low-k growth stimulated by the nongyrotropic alphas occurs at somewhat higher wavenumbers when all the protons are gyrotropic.

The dichromatic gyrophase distributions yield the dispersion shown in Fig. 4. Now the electrostatic eigenwave does not interact with the electromagnetic eigenmodes; its unmodified dispersion is given in Fig. 1.
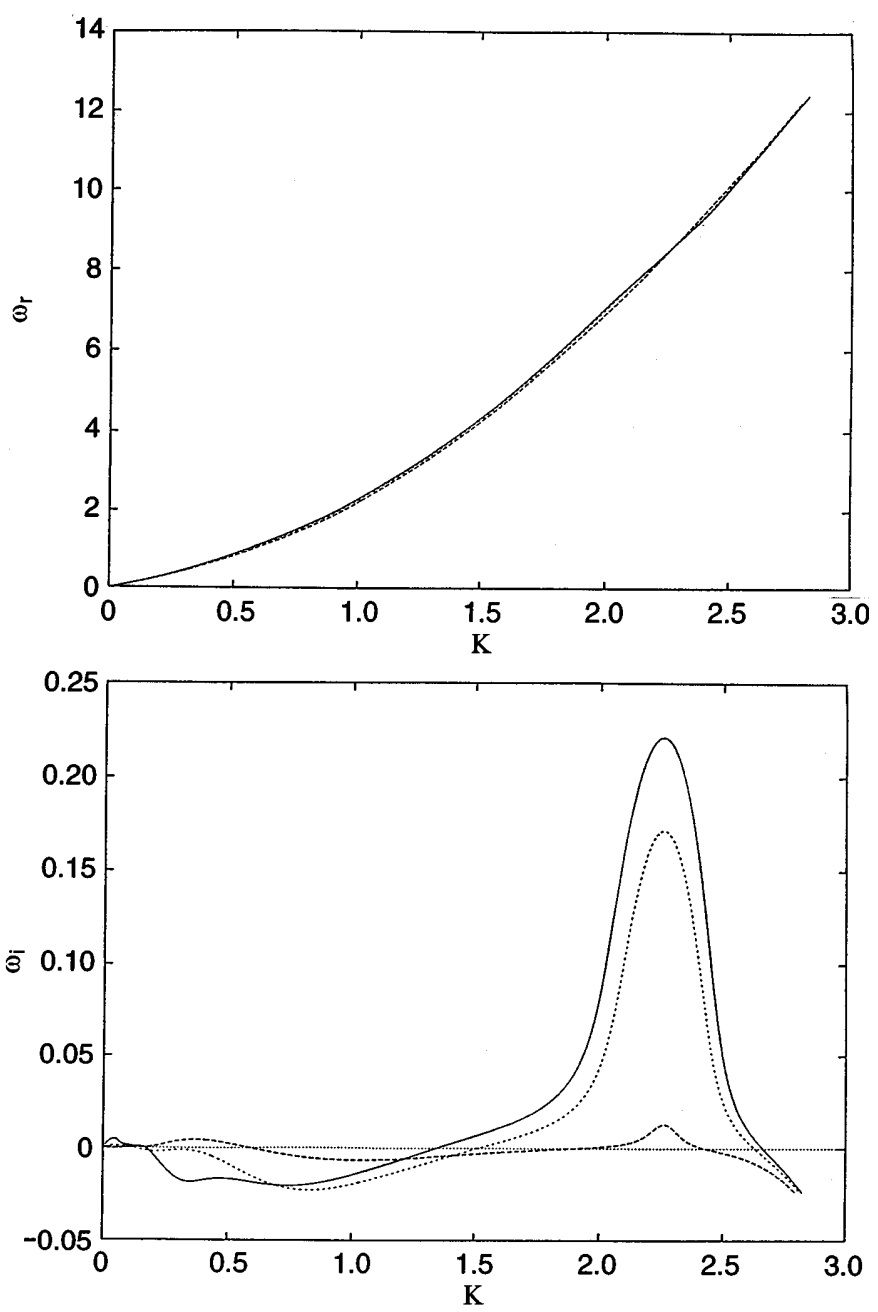

Fig. 3. Real and imaginary parallel dispersion of the reference magnetoplasma (unstable modes) for three situations where the gyrophase bunchings are monochromatic: (solid line) nongyrotropic protons and alpha particles, as in Fig. 2; (long dashed line) gyrotropic protons and nongyrotropic alpha particles, (short dashed line) gyrotropic alpha particles and nongyrotropic protons. Normalizations as in Fig. 1
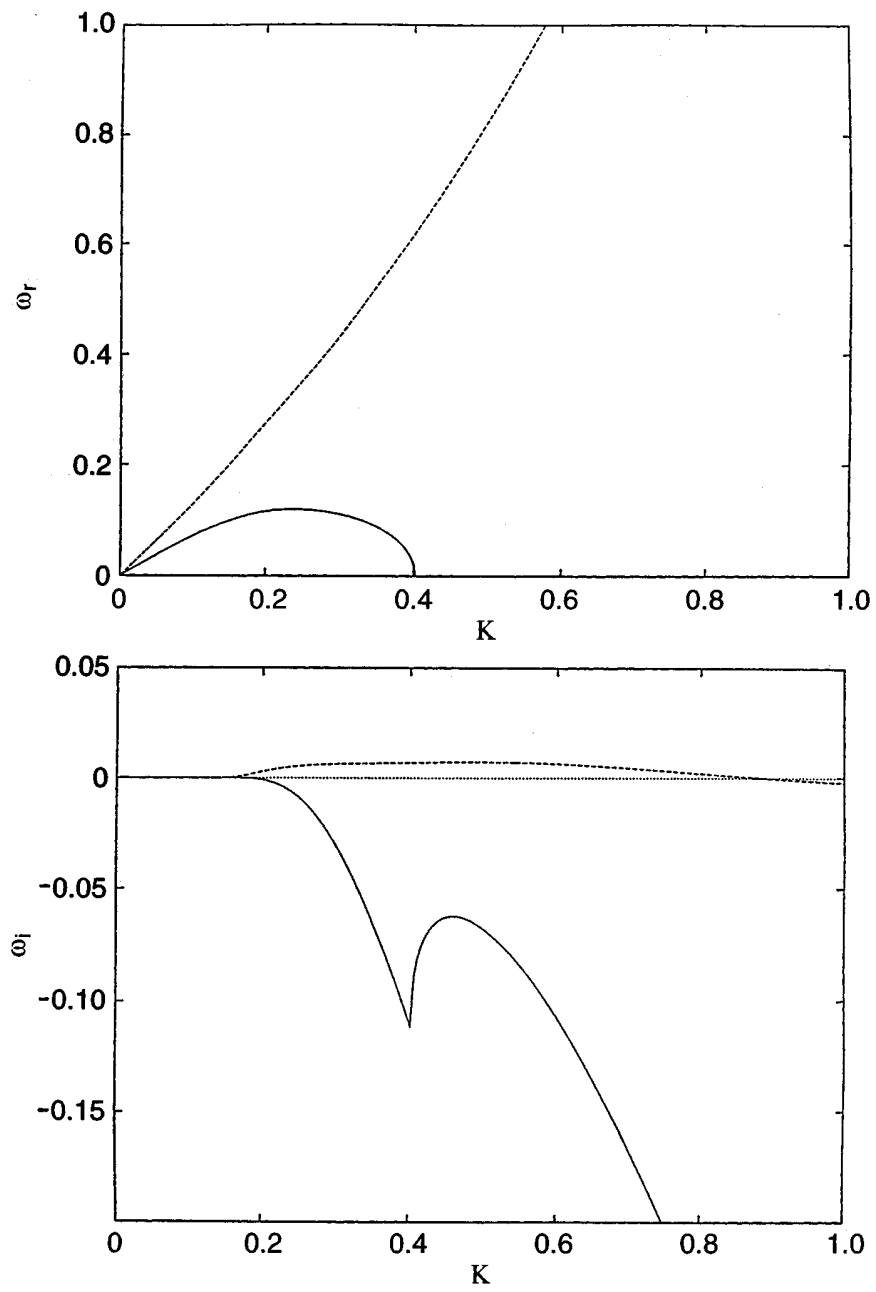

Fig. 4. Real and imaginary dispersion of the reference magnetoplasma when the unperturbed gyrophases of the protons and alpha particles are dichromatic. Normalizations as in Fig. 1.

The coupling between the left-hand and right-hand eigenmodes brought about by the introduction of gyrophase bunching also destabilizes the Maxwellian medium: one of the modes grows from $k v_{A} / \Omega_{p}=0.17$ to 0.88 with a maximum of $\omega_{i} / \Omega_{p}=0.0074$ at $k v_{A} / \Omega_{p}=0.49$. The other mode is stable but displays zero real frequency for large wavenumbers $\left(k v_{A} / \Omega_{p}>0.4\right)$; an increase (by a factor of 2, for example) of the densities of all the particle populations would bring about a purely growing nonoscillatory mode, as already found for one dichromatic species (Brinca and Romeiras, 1998).

The analysis presented in Appendix B suggests that the parallel wave growth found above for "external" SNG systems is strongly squelched when BGK-like "internal" sink terms are taken into consideration: studies on the stability of SNG environments require careful modeling of the (necessary) sources and sinks.

\section{Perpendicular propagation}

Oblique electromagnetic propagation in SNG magnetoplasmas with one phase bunched population was 
studied, for the cold plasma approximation, by Cao et al. (1998) and Motschmann and Glassmeier (1998); perpendicular electrostatic stability in the same environment with extreme gyrophase organizations (but without the restriction to cold plasmas) was studied by Romeiras and Brinca (1999). Investigations of perpendicular electrostatic propagation in TNG plasmas started with Eldridge (1970) for very weak nongyrotropies. All these approaches demonstrated that comprehensive analytical consideration of nongyrotropic effects on oblique (perpendicular, in particular) propagation is only feasible when adopting (sometimes drastic) simplifications. Recourse to numerical simulations of these environments is thus an appealing alternative. However, this tool has not yet devised a means of appropriately generating a SNG magnetoplasma: recycling techniques generate excessive noise that masks effects under observation. To date, simulation results of perpendicular propagation in nongyrotropic media are only available for TNG plasmas: they started with the work of Brinca et al. (1998) that demonstrated the growth of perpendicular electrostatic and extraordinary modes in (otherwise passive) magnetoplasmas with TNG gyrophase bunched electrons. Here, using the same KEMPO1 code, we shall extend the simulation model of this study to contemplate electrostatic perpendicular propagation in TNG media with two coexisting nongyrotropic ion populations.

\subsection{Model}

The envisaged neutral magnetoplasma has an ambient magnetic field aligned with the $y$ axis $\left(\varphi=\arctan v_{z} / v_{x}\right)$ and three isotropic, maxwellian particle populations: electrons, positrons $\left(m_{p}=m_{e}\right)$, and singly positively charged fictitious ions with $m_{f}=2 m_{e}$. The electrons are always gyrotropic while each ion population $\left(N_{p}=N_{f}=N_{e} / 2\right)$ can assume dichromatic gyrophase bunchings; their cyclotron frequencies satisfy $-\Omega_{e}=\Omega_{p}=2 \Omega_{f}$. (This unrealistic choice of ion species has to do with the penalization suffered by the kinetic simulations when trying to achieve appropriate temporal resolution in media with particles of very different masses but it does not hinder the illustration of the effects under analysis; hybrid codes should be able to handle realistic ion masses and qualitatively verify the observed phenomenology.) Now the ambient magnetic field is parallel to the $y$ axis, and the wavevector and (electric) wave field of the studied modes are aligned with the $x$ direction, with the relevant parameters adopted in the simulation runs listed in Table 1.

The results shown below arise from four basic simulation runs. The reference state is obtained with the three particle populations taken as gyrotropic; the system is necessarily stable because the nondrifting velocity distributions are isotropic maxwellians (run G). Run $\mathrm{F}$ takes a dichromatic gyrophase distribution for the fictitious ions (only the orientations of their perpendicular velocities are organized; in particular, their kinetic energies are unmodified) and maintains
Table 1. Simulation parameters

\begin{tabular}{lc}
\hline Parameter & Value \\
\hline Electron cyclotron frequency & -1 \\
Positron cyclotron frequency & 1 \\
Fictitious ion cyclotron frequency & 0.5 \\
Electron plasma frequency & 2.8 \\
Positron plasma frequency & 2.0 \\
Fictitious ion plasma frequency & 1.4 \\
Electron $(\|$ and $\perp$ ) thermal speed & 1 \\
Positron $(\|$ and $\perp$ ) thermal speed & 1 \\
Fictitious ion $(\|$ and $\perp$ ) thermal speed & 1 \\
Grid spacing & 0.25 \\
Time step & 0.02 \\
System length & 64 \\
Number of super electrons & 262144 \\
Number of super positrons & 262144 \\
Number of super fictitious ions & 262144 \\
\hline
\end{tabular}

gyrotropy for the remaining particle populations. Dichromatic gyrophases in the positrons and gyrotropy in the other species leads to run $\mathrm{P}$ and, finally, run PF introduces dichromatic gyrophases in the two ion populations keeping the electrons gyrotropic.

Normalizations in time and velocity are made with respect to $1 / \Omega_{p}$ and $v_{t p}$ (positron inverse cyclotron frequency and thermal speed), so that distance $(X)$, angular frequency $(\omega)$, and wavenumber $(K)$ are normalized to $v_{t p} / \Omega_{p}, \Omega_{p}$ and $\Omega_{p} / v_{t p}$, respectively; other plotted quantities below (electric field and electric energy density) have arbitrary units.

\subsection{Simulation results}

The temporal evolution of the electric field wavenumber spectra is shown in Fig. 5 for the four basic runs (from top to bottom: G, F, P, PF) at discrete times. The mild amplitude growth observed in runs $\mathrm{F}$ and $\mathrm{P}$ contrasts with the robust increase stimulated in run PF which also displays the narrower spectrum.

Figure 6 depicts the distribution of the perpendicular velocities of the nongyrotropic populations at the beginning and end of runs $\mathrm{F}, \mathrm{P}$, and PF. It is clear that the gyrophase diffusion observed in run PF is much more intense than the diffusion that could have been anticipated from the superposition of the diffusions obtained in runs $\mathrm{F}$ and $\mathrm{P}$.

The real dispersion $\omega_{r}(k)$ of the wave activity stimulated in the four basic runs and the corresponding temporal evolution of the wave electric energy densities are provided in Fig. 7. It is evident that (i) among the unstable nongyrotropic runs $(\mathrm{F}, \mathrm{P}$, and $\mathrm{PF}$ ) growth is much more intense when the two gyrophase bunched ion populations coexist (run PF) and (ii) the PF dispersion could have not been inferred from the "superposition" of the dispersions obtained for one nongyrotropic ion species medium (runs $\mathrm{F}$ and $\mathrm{P}$ ).

In retrospect, this observed synergic enhancement of wave growth due to the coexistence of two dichromatic (in general, nongyrotropic) ion populations whose 

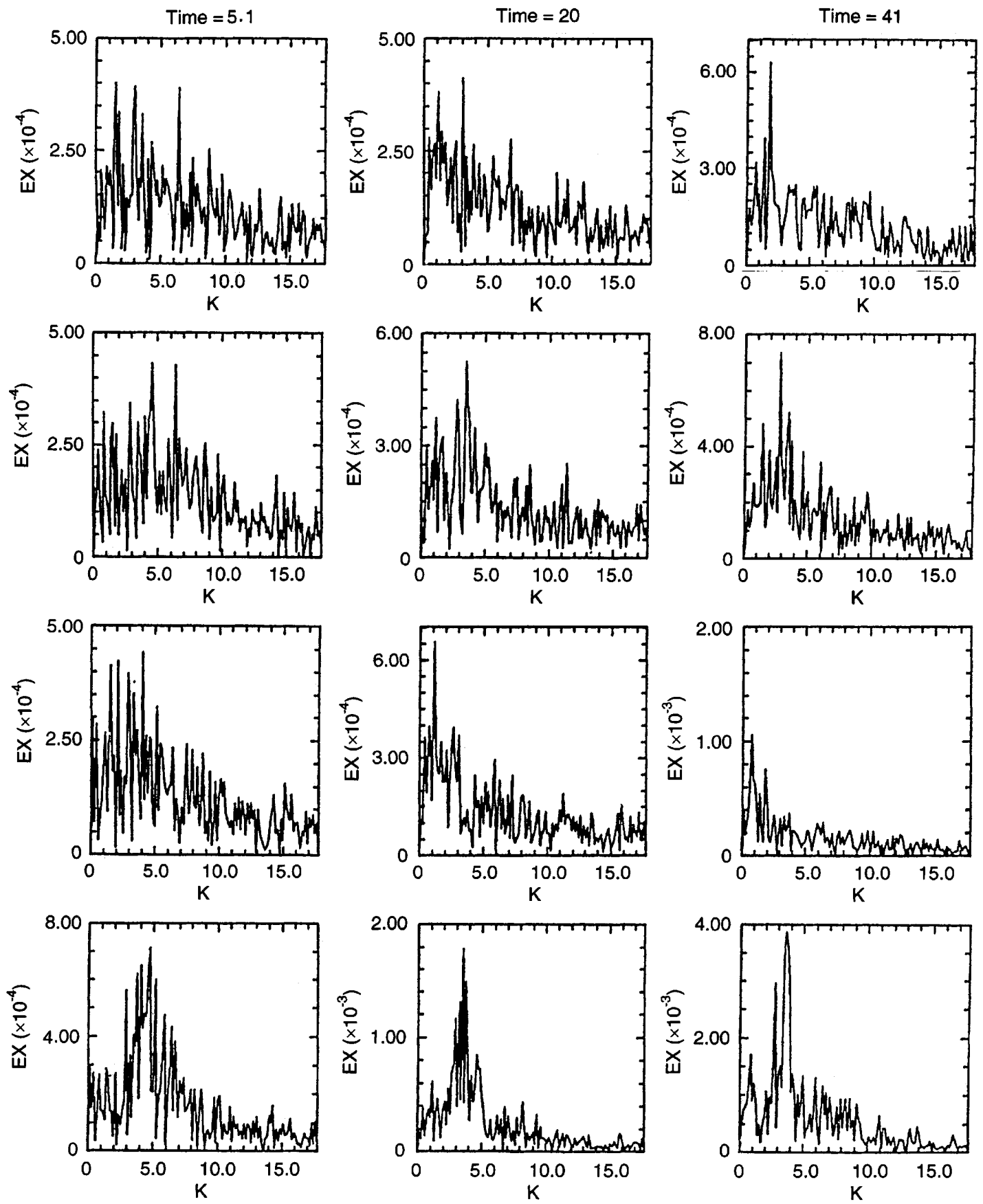

Fig. 5. Wavenumber spectra of the electric field at $t=5.1$ (left panel), $t=20$ (middle panel), and $t=41$ (right panel). Normalizations and runs as defined in the text

cyclotron frequencies are harmonic $(2: 1$ ratio in this case) could probably have been anticipated. An electrostatic wave along the $\mathrm{x}$ axis in order to grow from the free energy of a single dichromatic population must organize itself in $\omega$ and $k$ so that at certain time intervals can (on average) decelerate the gyrophase bunched particles; adding to this environment another (in this case) dichromatic species with a harmonic (for example, $2: 1)$ cyclotron frequency, it seems reasonable that the new particles can contribute to the growth of some of the previously excited modes. Indeed, the Brillouin diagrams on the left panel of Fig. 7 suggest that the dichromatic positrons were able to enhance the growth of some of the modes that had been excited by the dichromatic fictitious ions. An imperfect analogy with the underlying phenomenology is provided by the lighting effect obtained with two stroboscopic lamps of frequencies $f$ and $2 f$.

\section{Discussion}

This theoretical investigation on the stability of two coexisting nongyrotropic species shows that their effects 
TIME $=0.0$
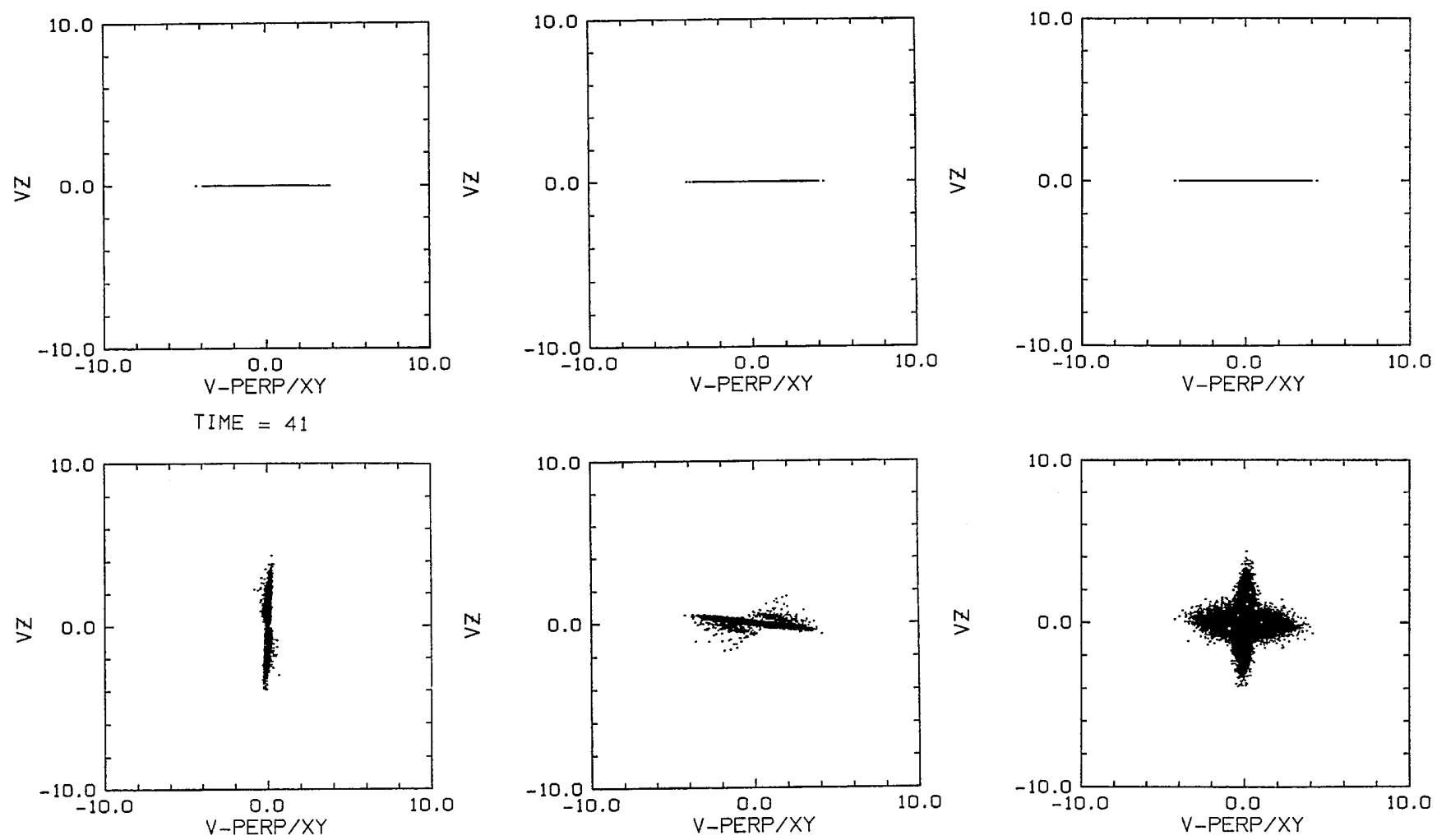

Fig. 6. Distributions of the perpendicular velocities of the nongyrotropic populations at the beginning $(t=0$ : upper panel) and end ( $t=41$ : lower panel) of the simulations (V-PERP/XY $=\mathrm{VX})$. From left to right: runs F, P, and PF. Velocity normalization defined in the text

on parallel propagation in SNG magnetoplasma can be qualitatively anticipated from the characteristics of media with (the same two) single gyrophase bunched populations: roughly, the two SNG species feed their own instabilities and their coexistence does not bring about new significant interactions. In contrast, the kinetic simulations carried out for perpendicular electrostatic propagation in TNG magnetoplasmas suggest the occurrence of very sizable synergic enhancements of wave growth and particle diffusion, in association with modifications of the complex wave dispersion, when the two nongyrotropic populations with harmonic cyclotron frequencies are made to coexist. These dissimilar effects for parallel and oblique (perpendicular, in particular) wave growth, bearing in mind that the free energy is provided by the gyrophase bunched particles, are compatible with the well-known characteristics of wave-particle interactions: parallel waves sense only the fundamental cyclotron resonances (and the Landau resonance for the longitudinal electric field) whereas oblique modes can resonate with (any order) cyclotron harmonics.

The extreme models adopted in the analysis (gyrophase organization, density of the nongyrotropic species and, in the perpendicular stability studies, the masses of the ions) are compatible with the objectives of this investigation. Here we are not concerned with specific plasma environments or the interpretation of wave activity observations in space. Rather, we illustrate basic physical effects originated in populations exhibiting gyrophase organization that, bearing in mind the prevalence of nongyrotropy in space plasmas and their proclivity to feed wave growth, might benefit the understanding of future field observations.

\section{Appendix A}

The matrix elements $m_{\eta v}$ of the SNG magnetoplasma under discussion with "external" source and sinks are given by

$$
\begin{aligned}
m_{-+}= & k^{2} c^{2}-\omega^{2}-\pi \omega \\
\times & \left\{\sum_{l=e, p, a} \omega_{p l}^{2} \int_{-\infty}^{\infty} \frac{\mathrm{d} v_{x}}{\omega-k v_{x}-\Omega_{l}}\right. \\
& \times \int_{0}^{\infty} \mathrm{d} v_{\perp} v_{\perp}^{2}\left[\left(1-\frac{k v_{x}}{\omega}\right) \frac{\partial F_{o l}}{\partial v_{\perp}}+\frac{k v_{\perp}}{\omega} \frac{\partial F_{o l}}{\partial v_{x}}\right] \\
& +\sum_{s=b, d} \omega_{p s}^{2} \int_{-\infty}^{\infty} \frac{\mathrm{d} v_{x}}{\omega-k v_{x}-\Omega_{s}} \int_{0}^{\infty} \mathrm{d} v_{\perp} v_{\perp}^{2} \\
& \left.\times\left[\left(1-\frac{k v_{x}}{\omega}\right) \frac{\partial G_{o s}}{\partial v_{\perp}}+\frac{k v_{\perp}}{\omega} \frac{\partial G_{o s}}{\partial v_{x}}\right] \phi_{o s}\right\}
\end{aligned}
$$



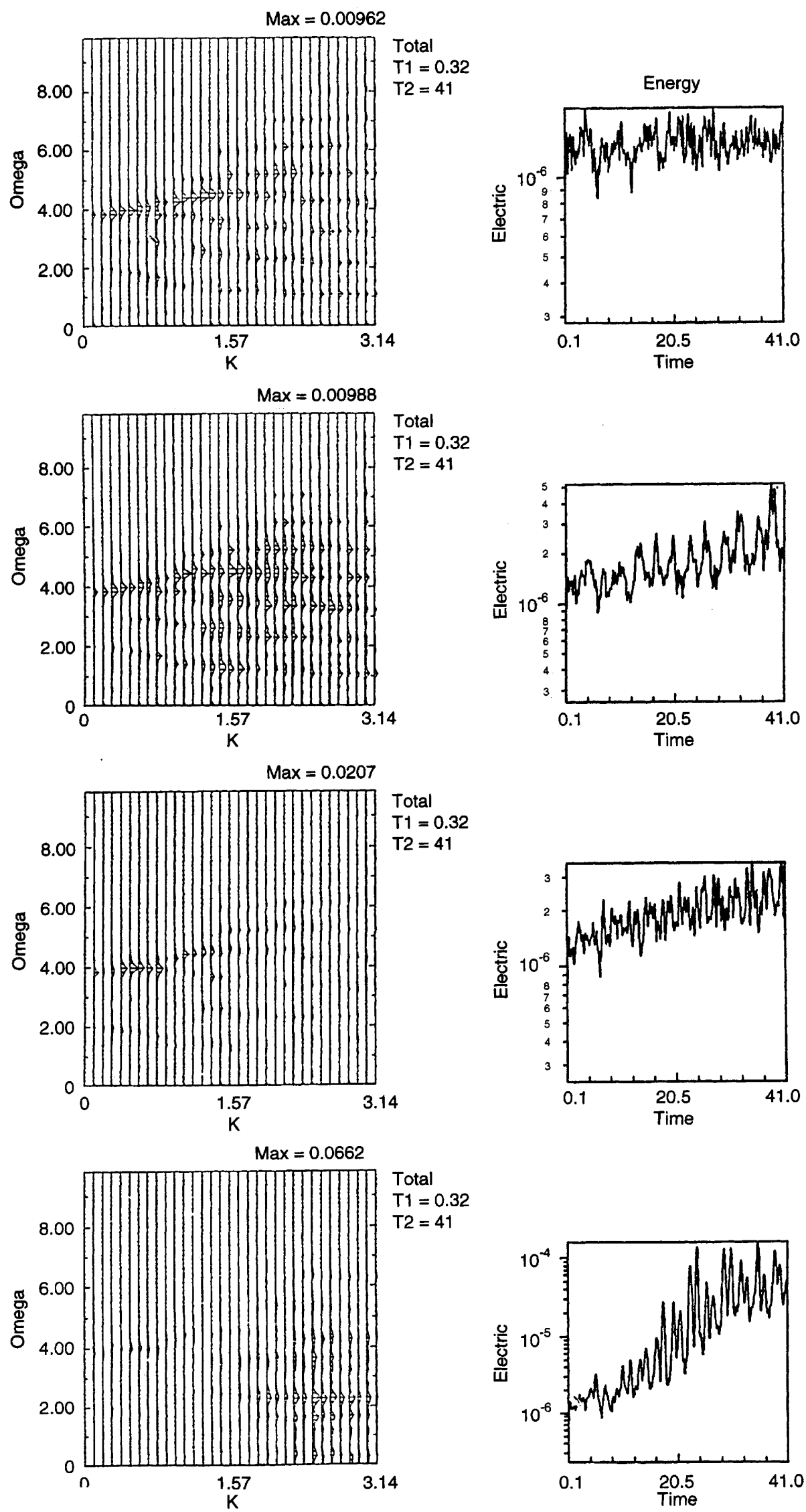

Fig. 7. Simulation results for the four basic runs: real dispersion diagram (left panel) and temporal evolution of the wave electric field energy density (right panel). From top to bottom: runs G, F, P, and $\mathrm{PF}$; energy density with arbitrary units 


$$
\begin{aligned}
& m_{+x}=-\pi \omega \sum_{s=b, d} \omega_{p s}^{2} \int_{-\infty}^{\infty} \frac{\mathrm{d} v_{x}}{\omega-k v_{x}-\Omega_{s}} \\
& \times \int_{0}^{\infty} \mathrm{d} v_{\perp} v_{\perp}^{2} \frac{\partial G_{o s}}{\partial v_{x}} \phi_{s(+1)} \\
& m_{+-}=-\pi \omega \sum_{s=b, d} \omega_{p s}^{2} \int_{-\infty}^{\infty} \frac{\mathrm{d} v_{x}}{\omega-k v_{x}-\Omega_{s}} \\
& \times \int_{0}^{\infty} \mathrm{d} v_{\perp} v_{\perp} 2\left(\frac{k v_{\perp}}{\omega} \frac{\partial G_{o s}}{\partial v_{x}}\right) \phi_{s(+2)} \\
& m_{x+}=\frac{2 \pi}{\omega} \sum_{s=b, d} \omega_{p s}^{2} \int_{-\infty}^{\infty} \frac{\mathrm{d} v_{x} v_{x}}{\omega-k v_{x}} \int_{0}^{\infty} \mathrm{d} v_{\perp} v_{\perp} \frac{k v_{\perp}}{\omega} \frac{\partial G_{o s}}{\partial v_{x}} \phi_{s(-1)} \\
& m_{x x}=1+\frac{2 \pi}{\omega}\left\{\sum_{l=e, p, a} \omega_{p l}^{2} \int_{-\infty}^{\infty} \frac{\mathrm{d} v_{x} v_{x}}{\omega-k v_{x}} \int_{0}^{\infty} \mathrm{d} v_{\perp} v_{\perp} \frac{\partial F_{o l}}{\partial v_{x}}\right. \\
& \left.+\sum_{s=b, d} \omega_{p s}^{2} \int_{-\infty}^{\infty} \frac{\mathrm{d} v_{x} v_{x}}{\omega-k v_{x}} \int_{0}^{\infty} \mathrm{d} v_{\perp} v_{\perp} \frac{\partial G_{o s}}{\partial v_{x}} \phi_{s o}\right\} \\
& m_{x-}=\frac{2 \pi}{\omega} \sum_{s=b, d} \omega_{p s}^{2} \int_{-\infty}^{\infty} \frac{\mathrm{d} v_{x} v_{x}}{\omega-k v_{x}} \int_{0}^{\infty} \mathrm{d} v_{\perp} v_{\perp} \frac{k v_{\perp}}{\omega} \frac{\partial G_{o s}}{\partial v_{x}} \phi_{s(+1)} \\
& m_{-+}=-\pi \omega \sum_{s=b, d} \omega_{p s}^{2} \int_{-\infty}^{\infty} \frac{\mathrm{d} v_{x}}{\omega-k v_{x}+\Omega_{s}} \\
& \times \int_{0}^{\infty} \mathrm{d} v_{\perp} v_{\perp}^{2}\left(\frac{k v_{\perp}}{\omega} \frac{\partial G_{O S}}{\partial v_{x}}\right) \phi_{S(-2)} \\
& m_{-x}=-\pi \omega \sum_{s=b, d} \omega_{p s}^{2} \int_{-\infty}^{\infty} \frac{\mathrm{d} v_{x}}{\omega-k v_{x}+\Omega_{s}} \\
& \times \int_{0}^{\infty} \mathrm{d} v_{\perp} v_{\perp}^{2} \frac{\partial G_{o s}}{\partial v_{x}} \phi_{s(-1)} \\
& m_{--}=k^{2} c^{2}-\omega^{2}-\pi \omega \\
& \times\left\{\sum_{l=e, p, a} \omega_{p l}^{2} \int_{-\infty}^{\infty} \frac{\mathrm{d} v_{x}}{\omega-k v_{x}+\Omega_{l}}\right. \\
& \times \int_{0}^{\infty} \mathrm{d} v_{\perp} v_{\perp}^{2}\left[\left(1-\frac{k v_{x}}{\omega}\right) \frac{\partial F_{o l}}{\partial v_{\perp}}+\frac{k v_{\perp}}{\omega} \frac{\partial F_{o l}}{\partial v_{x}}\right] \\
& +\sum_{s=b, d} \omega_{p s}^{2} \int_{-\infty}^{\infty} \frac{\mathrm{d} v_{x}}{\omega-k v_{x}+\Omega_{s}} \int_{0}^{\infty} \mathrm{d} v_{\perp} v_{\perp}^{2} \\
& \left.\times\left[\left(1-\frac{k v_{x}}{\omega}\right) \frac{\partial G_{o s}}{\partial v_{\perp}}+\frac{k v_{\perp}}{\omega} \frac{\partial G_{o s}}{\partial v_{x}}\right] \phi_{s o}\right\}
\end{aligned}
$$

\section{Appendix B}

The unperturbed distribution functions of the nongyrotropic species $(s=b, d)$ satisfy the Vlasov equation, possibly with source $(S)$ and sink $(L)$ terms on the rhs,

$$
\left(\frac{\partial}{\partial t}+\mathbf{v} \cdot \frac{\partial}{\partial \mathbf{r}}-\Omega_{s} \frac{\partial}{\partial \varphi}\right) F_{s o}=S_{s}-L_{s}
$$

In closed phase spaces $\left(S_{s}=L_{s}=0\right)$, it is clear that homogeneous unperturbed distributions must be timevarying (TNG). Consideration of open phase spaces allows for the existence of homogeneous and stationary (SNG) solutions that depend on the choice of the source and sink terms, as exemplified for instance in Motschmann et al. (1997). The nature of these terms strongly influences the ensuing analysis of the dispersion and stability of the nongyrotropic ambient.

If it is acceptable to assume that the sources (e.g. models of cometary newborn ions) and sinks (e.g. preexisting turbulence that brings about diffusion in phase space) are "external" to the system under analysis, i.e. they are not affected by the small amplitude wave perturbations considered in the linearization of the system $\left(S_{s 1}=L_{s 1}=0\right)$, then they determine the unperturbed nongyrotropic distributions, $F_{S o}$, but do not intervene on the linear dispersion and stability of the medium: we followed this approach above. To date, all the available studies of SNG behavior have, explicitly or implicitly, adopted the hypothesis of "external" sources and sinks.

Consideration of the dispersion and stability effects of "internal" terms requires their specification and can strongly modify the results obtained with the "external" model. To illustrate this point, we choose simple loss terms of the BGK-type invoked by Cao et al. (1995) and Motschmann et al. (1997), $L_{s}=F_{s} / \tau_{s}$ but, in contrast to these authors who assumed implicitly to have $L_{s 1}=0$, we admit "internal" losses so that $L_{s 1}=F_{s 1} / \tau_{s}$.

The "external" source terms, as adopted by the previous authors, are such that

$\Phi_{s}(\varphi)=\frac{e^{\varphi / \Delta_{s}}}{\Delta_{s}\left(1-e^{-2 \pi / \Delta_{s}}\right)}$

in the interval $(-2 \pi<\varphi \leq 0)$ and $2 \pi$-periodic elsewhere, with $\Delta_{s}=\Omega_{s} \tau_{s}$. The relevant Fourier coefficients become

$$
\begin{aligned}
& \phi_{s(+1)}=\phi_{s(-1)}^{*}=\frac{1-i \Delta_{s}}{2 \pi\left(1+\Delta_{s}^{2}\right)}, \\
& \phi_{s(+2)}=\phi_{s(-2)}^{*}=\frac{1-i 2 \Delta_{s}}{2 \pi\left(1+4 \Delta_{s}^{2}\right)} .
\end{aligned}
$$

and the matrix elements $m_{\eta v}$ for this new nongyrotropic environment with Maxwellian velocity distributions in $v_{\perp}$ and $v_{x}$ are given by 


$$
\begin{aligned}
& m_{++}=k^{2} c^{2}-\omega^{2}-\sum_{l=e, p, a} \omega_{p l}^{2} \\
& \times\left[\frac{\omega}{k v_{t l}}\left(1-\frac{k V_{D l}}{\omega}\right) Z\left(\xi_{l+}\right)\right. \\
& \left.-\frac{1}{2}\left(A_{l}-1\right) Z^{\prime}\left(\xi_{l+}\right)\right]-\sum_{s=b, d} \omega_{p s}^{2} \\
& \times\left[\frac{\omega}{k v_{t s}}\left(1-\frac{k V_{D s}}{\omega}\right) Z\left(\tilde{\xi}_{s+}\right)-\frac{1}{2}\left(A_{s}-1\right) Z^{\prime}\left(\tilde{\xi}_{s+}\right)\right] \\
& m_{+x}=\frac{\pi^{3 / 2}}{2} \sum_{s=b, d} \sqrt{A_{s}} \frac{\omega}{k v_{t s}} \omega_{p s}^{2} \phi_{s(+1)} Z^{\prime}\left(\tilde{\xi}_{s+}\right) \\
& m_{+-}=\pi \sum_{s=b, d} A_{s} \omega_{p s}^{2} \phi_{s(+2)} Z^{\prime}\left(\tilde{\xi}_{s+}\right) \\
& m_{x+}=-\pi^{3 / 2} \sum_{s=b, d} \sqrt{A_{s}}\left(\frac{\omega_{p s}}{\omega}\right)^{2} \frac{\tilde{\omega}_{s}}{k v_{t s}} \phi_{s(-1)} Z^{\prime}\left(\xi_{s x}\right) \\
& m_{x x}=1-\sum_{l=e, p, a}\left(\frac{\omega_{p l}}{k v_{t l}}\right)^{2} Z^{\prime}\left(\xi_{l x}\right) \\
& -\sum_{s=b, d} \frac{\tilde{\omega}_{s}}{\omega}\left(\frac{\omega_{p s}}{k v_{t s}}\right)^{2} Z^{\prime}\left(\tilde{\xi}_{s x}\right) \\
& m_{x-}=-\pi^{3 / 2} \sum_{s=b, d} \sqrt{A_{s}}\left(\frac{\omega_{p s}}{\omega}\right)^{2} \frac{\tilde{\omega}_{s}}{k v_{t s}} \phi_{s(+1)} Z^{\prime}\left(\tilde{\xi}_{s x}\right) \\
& m_{-+}=\pi \sum_{s=b, d} A_{s} \omega_{p s}^{2} \phi_{s(-2)} Z^{\prime}\left(\tilde{\xi}_{s-}\right) \\
& m_{-x}=\frac{\pi^{3 / 2}}{2} \sum_{s=b, d} \sqrt{A_{s}} \frac{\omega}{k v_{t s}} \omega_{p s}^{2} \phi_{s(-1)} Z^{\prime}\left(\tilde{\xi}_{s-}\right) \\
& m_{--}=k^{2} c^{2}-\omega^{2}-\sum_{l=e, p, a} \omega_{p l}^{2} \\
& \times\left[\frac{\omega}{k v_{t l}}\left(1-\frac{k V_{D l}}{\omega}\right) Z\left(\xi_{l-}\right)\right. \\
& \left.-\frac{1}{2}\left(A_{l}-1\right) Z^{\prime}\left(\xi_{l-}\right)\right]-\sum_{s=b, d} \omega_{p s}^{2} \\
& \times\left[\frac{\omega}{k v_{t s}}\left(1-\frac{k V_{D s}}{\omega}\right) Z\left(\tilde{\xi}_{s-}\right)-\frac{1}{2}\left(A_{s}-1\right) Z^{\prime}\left(\tilde{\xi}_{s-}\right)\right],
\end{aligned}
$$

where

$\tilde{\xi}_{s \pm}=\frac{\tilde{\omega}_{s}-k V_{D s} \mp \Omega_{s}}{k v_{t s}}, \quad \tilde{\xi}_{s x}=\frac{\tilde{\omega}_{s}-k V_{D s}}{k v_{t s}}$,

and $\tilde{\omega}_{s}=\omega+i / \tau_{s}$.

Going back to the SNG medium underlying the results for parallel propagation shown in Fig. 2, but now assuming the nongyrotropic proton and alpha particle populations to have gyrophase distributions $\Phi_{s}(\varphi)$ associated with "internal" loss terms as above, and using $\tau_{b}=\tau_{d}$ in the solution of the new modified dispersion equation, we realize that the most unstable choice $\left(\tau_{b} \Omega_{p}=\tau_{d} \Omega_{p}=2.3\right)$ is only barely able to bring about wave growth in one hybrid mode (positive growth rate in the band $2.76<k v_{A} / \Omega_{p}<2.87$, with a maximum of $\left.\omega_{i} / \Omega_{p}=0.0019\right)$.

The adopted "internal" loss term thus strongly squelches the instability of the system: while large $\tau_{s}$ generates distributions approaching gyrotropy and hence with Fourier coefficients $\phi_{s n}$ of small magnitude, small $\tau_{s}$ do bring about robust gyrophase bunching but introduce (the equivalent of) large damping rates in the contributions of the nongyrotropic species (arising from the perturbed Vlasov equation) to the wave dispersion. We stress, however, that this "internal" loss model is rather crude and inconsistent. Indeed, the decrease in the number of nongyrotropic particles of species $s$ (brought about by gyrophase mixing) should coexist with a corresponding increase in the number of gyrotropic particles of the same species (meaning that a source term should be added to the Vlasov equation of this gyrotropic population).

These results draw attention to the importance of adequately modeling the source and sink terms associated with the SNG species: further research on the genesis of gyrophase bunching in open phase spaces is warranted.

Acknowledgements. One of the authors (A.L.B.) gratefully acknowledges very helpful discussions with Gerard Belmont and the comprehensive access to the KEMPO1 code provided by Y. Omura and H. Matsumoto. We thank the members of the Nongyrotropy in Space Plasmas project for the continued support. This work was funded in part by FEDER and PRAXIS XXI.

Topical Editor K.-H. Glassmeier thanks G. Belmont and A. Coates for their help in evaluating this paper.

\section{References}

Astudillo, H. F., S. Livi, E. Marsch, and H. Rosenbauer, Evidence for nongyrotropic alpha particle and proton distribution functions: TAUS solar wind measurements, J. Geophys. Res., 101, 24423-24432, 1996.

Brinca, A. L., and F. J. Romeiras, On the stability of stationary nongyrotropic distribution functions: Coupling and purely growing waves, J. Geophys. Res., 103, 9275-9284, 1998.

Brinca, A. L., Y. Omura, and H. Matsumoto, Stability of prependicular propagation in time-varying nongyrotropic plasmas: Simulations, J. Geophys. Res., 103, 29493-29503, 1998.

Cao, J. B., C. Mazelle, G. Belmont, and H. Reme, Nongyrotropy of heavy newborn ions at comet Grigg-Skjellerup and corresponding instability, J. Geophys. Res., 100, 23379-23388, 1995.

Cao, J. B., C. Mazelle, G. Belmont, and H. Reme, Oblique ring instability driven by nongyrotropic ions: Application to observations at comet Grigg-Skjellerup, J. Geophys. Res., 103, 20552067, 1998.

Eldridge, O., Coupling of electrostatic waves in a nongyrotropic plasma, Phys. Fluids, 13, 1791-1794, 1970.

Mann, G., P. Hackenberg, and E. Marsch, Linear mode analysis in multi-ion plasmas, J. Plasma Phys., 58, 205-221, 1997.

Motschmann, U., and K.-H. Glaßmeier, Dispersion and wave excitation in nongyrotropic plasmas, J. Plasma Phys., 60, 111132, 1998.

Motschmann, U., H. Kafemann, and M. Scholer, Nongyrotropy in magnetoplasmas: Simulation of wave excitation and phasespace diffusion, Ann. Geophys., 15, 603-613, 1997.

Romeiras, F. J., and A.L. Brinca, On the stability of perpendicular electrostatic modes in stationary nongyrotropic plasmas, J. Geophys. Res., 104, 12407-12413, 1999.

Sudan, R. N., Growing waves in a nongyrotropic plasma, Phys. Fluids, 8, 1915-1918, 1965. 